\title{
Improvement of thermomechanical full-field analysis of metallic polycrystals using crystallographic data
}

\author{
Rian Seghir ${ }^{1,2,3}$, Jean-François Witz $^{1,2}$, Eric Charkaluk ${ }^{1,2,3, a}$ \\ AND Philippe DUfRÉNOY ${ }^{1,2,4}$ \\ 1 Univ. Lille Nord de France, 59000 Lille, France \\ 2 CNRS, UMR 8107, 59650 Villeneuve d'Ascq, France \\ 3 ECLille, LML, 59650 Villeneuve d'Ascq, France \\ 4 Univ. Lille1, Polytech'Lille, LML, 59650 Villeneuve d'Ascq, France
}

Received 10 February 2012, Accepted 20 November 2012

\begin{abstract}
This paper is based on additional treatments of the experimental results obtained by L. Bodelot, L. Sabatier, E. Charkaluk, P. Dufrénoy [Experimental setup for fully coupled kinematic and thermal measurements at the microstructure scale of an AISI $316 \mathrm{l}$ steel, Mater. Sci. Eng. A 501 (2009) 52-60]. In order to perform inter- and intragranular thermomechanical analyses in a metallic polycrystal at the grain scale, a crystallography-based projection technique of the thermal and displacement fields on a polynomial basis is proposed. It enables intragranular coupled analysis of strain and temperature full-field data consistent with the plastic slip activation observed on specimen surface after the test.
\end{abstract}

Key words: Infrared thermography / digital image correlation / microstructure / EBSD / strain localization

\begin{abstract}
Résumé - Amélioration de l'analyse de champs thermomécaniques de polycristaux métalliques à l'aide d'informations cristallographiques. Cet article est basé sur des traitements complémentaires des résultats expérimentaux présentés dans L. Bodelot, L. Sabatier, E. Charkaluk, P. Dufrénoy [Experimental setup for fully coupled kinematic and thermal measurements at the microstructure scale of an AISI 3161 steel, Mater. Sci. Eng. A 501 (2009) 52-60]. En vue de réaliser des analyses thermomécaniques inter- et intragranulaires à l'échelle des grains d'un polycristal métallique, une technique de projection, basée sur la cristallographie, des champs de température et de déplacement sur une base polynomiale est proposée. Cette technique permet des analyses couplées des champs de déformation et de température cohérentes avec l'activation du glissement plastique observée en surface de l'échantillon après essai.
\end{abstract}

Mots clés : Thermographie infrarouge / corrélation d'images numériques / microstructure / EBSD / localisation de la déformation

\section{Introduction}

During the mechanical loading of metallic polycrystals, the diversity of grain orientation leads to inhomogeneous deformation and results in local plasticity. These aspects have been studied and modeled for many decades and one can cite, for example, the pioneering work of Sachs and Ver [1], Schmid [2], Taylor [3]. These observations are extensively described, synthesized and modeled in the Cottrell's well-known book [4]. The clear observation of an inhomogeneous deformation field in

\footnotetext{
${ }^{a}$ Corresponding author:

eric.charkaluk@univ-lille1.fr
}

polycrystals has been particularly emphasized by Barrett et al. [5] and Boas et al. [6]. During deformation, this local and then global plasticity triggers a dissipation and a temperature increase in the specimen, a phenomenon that was studied very early by Taylor and co-workers [7-9] using global calorimetric measurements. It is well-known that during cold-working, most of the plastic work reappears in the form of heat, whereas a certain proportion remains latent in the material and is associated with microstructure changes, including mainly dislocation elastic energy. An extensive review of the early work within this field was published by Bever et al. [10]. Since the 80 's, many attempts have been made to access energy 
balances using infrared radiometers or cameras [11-14] to move from global to full-field measurements. Since it has been shown that the material class and the microstructure have notable effects on this energy balance $[15,16]$, estimating it at the grain scale becomes an interesting challenge.

However, these deformation processes lead to very low temperatures and occur at a very local scale, thus making any experimental measurement of stored energy laborious and complicated. As a result, to date, very few authors have attempted to achieve an energy balance at the microstructure scale of a polycrystal using full-field measurements combined with the heat equation. Preliminary steps were carried out on oligocrystals ( $<10$ grains) $[17,18]$. To our knowledge, the first attempt on polycrystals (hundreds or thousands of grains) based on a kinematic full-field measurement combined with a thermal measurement can be attributed to Bodelot et al. [19] who used an original experimental device [20] on an overquenched A316L austenitic stainless steel at a low strain level (i.e., <10\%) under monotonic tensile loading.

However, in [20], a clear connection of this energy balance with the microstructure was not demonstrated. The main reason is the spatial resolution of the full-field measurements, the coupling between Digital Image Correlation (DIC) and Infrared Thermography (IRT) and the noisy data obtained. Even if DIC results have already been obtained at the grain scales of polycrystals [21-24] under monotonic or cyclic loadings, these DIC measurements are generally taken using scanning electronic microscopes, because of the desired spatial resolutions. This is currently incompatible with simultaneous IRT measurements. So, new techniques have to be developed in order to achieve such DIC and IRT coupled measurements with high time and spatial resolutions. In this aim, this paper proposes specific treatments improved by crystallographic data of Bodelot's initial data [20].

The paper is divided into three parts. In the first one, the main characteristics of the experimental data of Bodelot et al. [20] are summarized and the initial thermomechanical fields are presented. Then the principle of a crystallography-based projection of these initial data on the microstructure using a polynomial basis is detailed. The fields obtained are then compared to the initial results. Finally, granular analyses are proposed showing the pertinence of such data treatment.

\section{Experimental data}

\subsection{Fully coupled measurements of kinematic and thermal fields}

The experimental set-up presented in this section has been developed in Bodelot's publications $[19,20]$. An original experimental setup was proposed, able to measure, in the same location and at the same time, kinematic and thermal fields at the microstructural scale on flat samples. The main features are described in [20]. The material studied is an AISI 316L austenitic stainless steel.
Dog-bone-shaped flat samples, $2 \mathrm{~mm}$ thick, were heattreated under air for $2 \mathrm{~h}$ at $1200{ }^{\circ} \mathrm{C}$ and immediately water-quenched. A displacement-controlled monotonic tensile test was applied to the sample with a constant strain rate of $5 \times 10^{-3} \mathrm{~s}^{-1}$ [19]. Compared to [19,20], all the treatments were completely redone (DIC, thermal calibration, emissivity map, Lagrangian thermography) but only the main characteristics are summarized here.

Strain fields are obtained using DIC performed with Correli [25] on images of the deforming sample covered with a speckle pattern. The spatial resolution of the kinematic fields (displacements, strains) is $104 \mu \mathrm{m}$ $\times 104 \mu \mathrm{m}$ after performing DIC on $16 \times 16$ pixel subsets of the images; the precision on displacement values is $4 \times 10^{2} \mu \mathrm{m}$ and the recording frequency is $7 \mathrm{~Hz}$. Temperature fields are obtained by IRT (Infrared Thermography) at a recording frequency of $140 \mathrm{~Hz}$, with a spatial resolution of $90 \mu \mathrm{m} \times 90 \mu \mathrm{m}$ for the thermal fields. This resolution corresponds to a smoothing operation on a $3 \times 3$ subset of $30 \mu \mathrm{m} \times 30 \mu \mathrm{m}$ pixels in order to improve the thermal resolution [26]. In this study, the link between the infrared radiation received by the camera and the temperature of the object is established with a blackbody through a calibration procedure applied to each detector of the camera resulting to a thermal resolution of $0.03{ }^{\circ} \mathrm{C}$.

Since the microstructure of a polycrystal is generally different from one face or location to the other on a flat specimen, the same area of the sample has to be observed simultaneously by both CCD and IRT cameras. To achieve such a fully coupled observation of the same zone, a dichroic mirror was placed in front of the sample, making a $45^{\circ}$-angle with the normal to its surface (see in [20]). Given its filtering properties, the dichroic mirror transmits the infrared radiation towards the infrared camera, located in front of the sample and reflects the rest of the radiation, including the visible radiation, to the CCD camera which is perpendicular to the normal of the sample. To take coupled measurements a special coating was designed and applied on the sample, simultaneously meeting requirements of both measurement techniques with the magnifications used. Finally, the DIC displacement data allowed tracking the displacement of material points in front of the infrared array and then expressing the thermal fields in the undeformed configuration. Therefore, the data is fully coupled insofar as both fields correspond to the same zone, at the same time and are given in the undeformed configuration. Then these fully coupled measurements at the microstructure scale were taken during the monotonic test, providing kinematic and thermal fields. The fields studied correspond to a $5 \mathrm{~mm} \times 5 \mathrm{~mm}$ area in the center of the specimen. After all these treatments, these fields will be denoted as initial fields.

\subsection{Microstructural analysis}

Complementary to these kinematic and thermal initial full-fields, Electron Back-Scattered Diffraction (EBSD) 


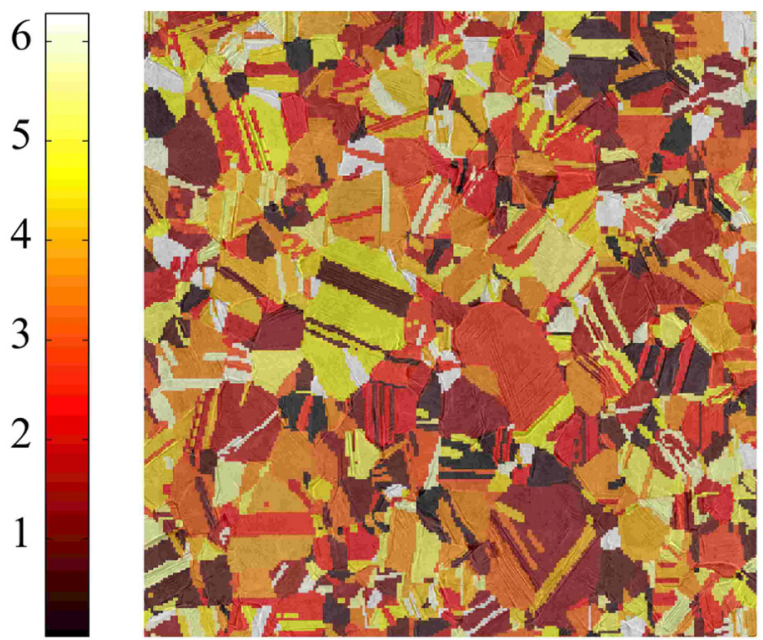

Fig. 1. First angle $\phi_{1}$ of the triplet $\left(\phi_{1}, \Phi, \phi_{2}\right)$ provided by the EBSD analysis (in rad), in the global frame of the analyzed area. The EBSD is superimposed on the micrography of the specimen after the test presented in Figure 2.

analysis was performed within a Jeol 6100 scanning electron microscope with a conventional electron gun operating at $25 \mathrm{kV}$; the probe current was on the order of $1 \mathrm{nA}$. Analyses were performed with OIM software provided by TSL with a pitch of $20 \mu \mathrm{m}$.

Figure 1 presents the EBSD field of the 1st angle $\phi_{1}$ of the triplet $\left(\phi_{1}, \Phi, \phi_{2}\right)$, which characterizes the 3D orientation of material points in the global frame of the analyzed area after the test. This field presents the mean grain orientation per grain.

The EBSD analysis shows that, in the $5 \mathrm{~mm} \times$ $5 \mathrm{~mm}$ central area, the microstructure is composed of 1776 grains with a $118 \mu \mathrm{m}$ mean grain size based on diameter approximation. Thus, the mean grain size of the tested sample matches the spatial resolution capabilities of both measurement techniques. However, note that $60.7 \%$ of the grains have a diameter lower than $100 \mu \mathrm{m}$, but this only represents $15.1 \%$ of the studied area. Then, more than $84 \%$ of the zone of interest is suitable for fullfield measurements analysis in order to observe heterogeneities. More particularly, approximatively 20 grains possess a grain size greater than $400 \mu \mathrm{m}$, which enables an intragranular investigation.

Figure 2 presents a micrography of the $5 \mathrm{~mm} \times 5 \mathrm{~mm}$ central area after the test. One can distinguish zones with many slip bands corresponding to a high intragranular plastic activity during the tensile loading and others, at the grain or twin boundaries, that exhibit large discontinuities corresponding to intergranular activity.

In order to analyze the strain and temperature evolutions in this $5 \mathrm{~mm} \times 5 \mathrm{~mm}$ central area, initial displacement fields are generally derived through a direct finite difference scheme in order to calculate the corresponding strain fields. This strain field and the temperature field obtained at the end of the tensile test are presented in Figures 3 and 4 . One can observe relatively noisy fields

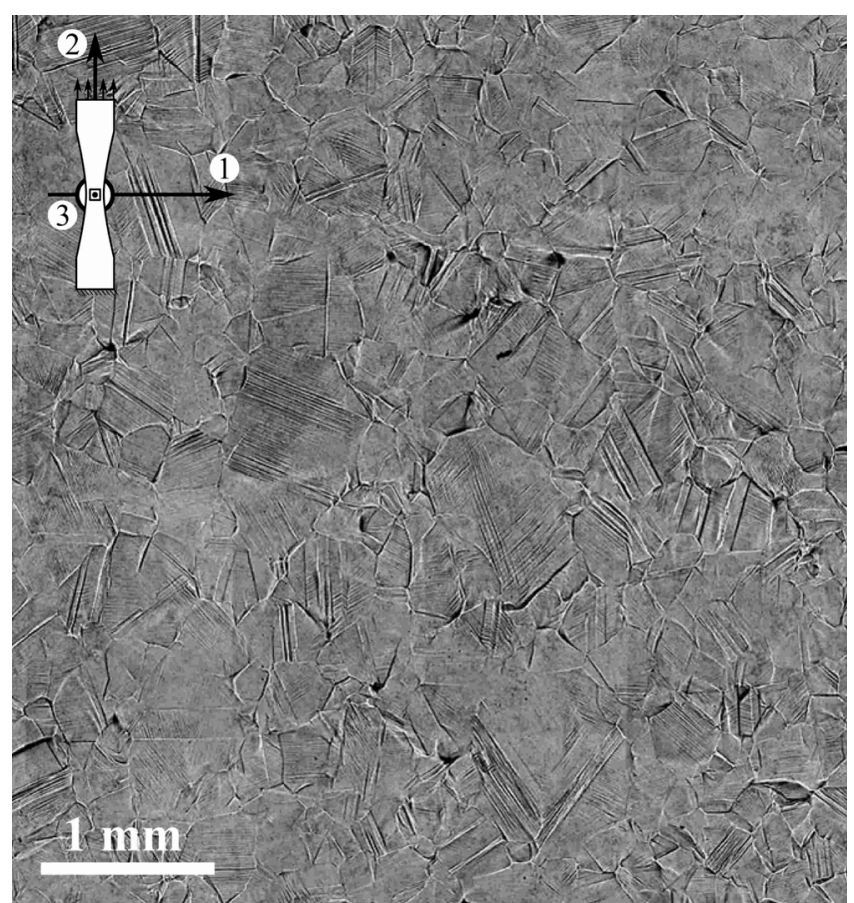

Fig. 2. Micrography of the $5 \mathrm{~mm} \times 5 \mathrm{~mm}$ central area after the test with the specimen frame orientations.

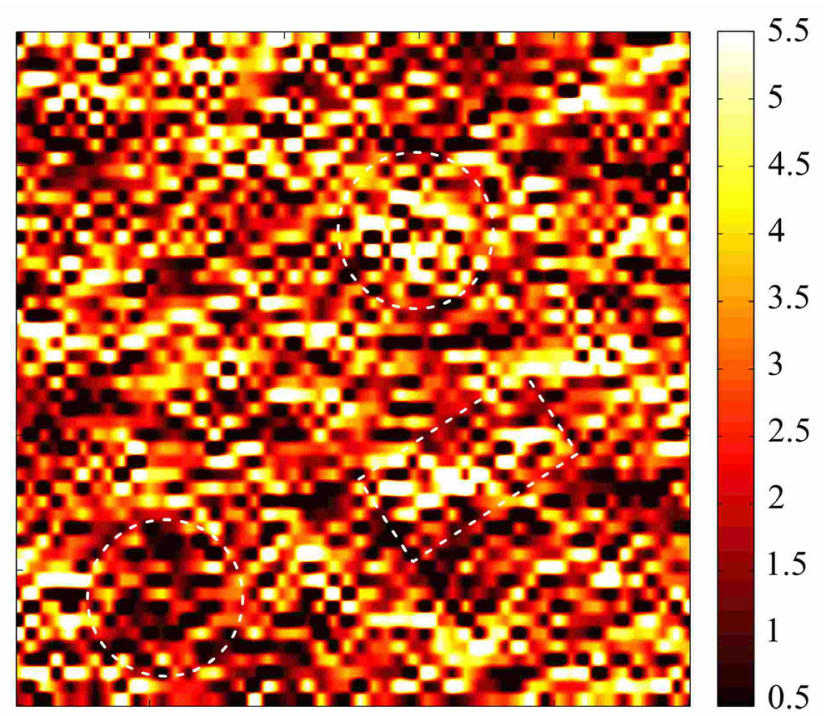

Fig. 3. Axial strain field (in \%) in the $5 \mathrm{~mm} \times 5 \mathrm{~mm}$ central area at the end of the test obtained from the initial displacement field by a direct finite difference scheme.

due to the derivative operators for the strain fields and to intrinsically noisy data due to the different sensor dynamics, as well as the variations of the observed signal, the environment, etc. Therefore, even if qualitative analysis is possible in order to relate field time evolution $[19,20]$ with the microstructure, these analyses remain a difficult task. Different ways could be considered to improve data treatment: (1) reduction of the spatial noise using a smoothing technique in the Fourier space [27]; (2) the approximation 


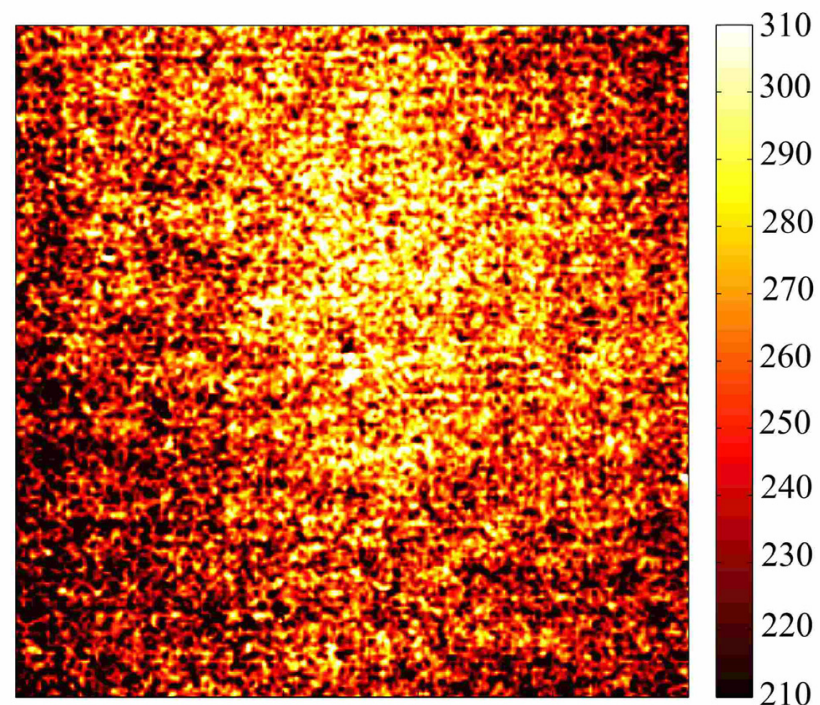

Fig. 4. Initial thermal field (in $\mathrm{mK}$ ) in the $5 \mathrm{~mm} \times 5 \mathrm{~mm}$ central area at the end of the test.

of the strain tensor within an $\Omega$ domain using a surface integral on $\partial \Omega$ [21], where $\partial \Omega$ describes the boundaries of the domain $\Omega$ : in this particular case, when $\Omega$ is the domain occupied by a given grain of the microstructure, the surface integral on $\partial \Omega$ represents the mean strain over this grain; (3) a smoothing operation on the displacement and temperature fields over a rectangular window by least square minimization of a high-order polynomial [28]. In these different techniques, one can note that the highfrequency parts of information are also eliminated; this is particularly harmful in the case of highly heterogeneous fields.

\section{Numerical projection of fields on grains}

In this paper, using the complementary EBSD field is proposed in order to project the initial measurements on the microstructure. In fact, the EBSD data provide the boundaries of each grain within the central area analyzed. The grain (or twin) boundary network constitutes a natural partition between intra- and intergranular domains and it is well known that grain and twin boundaries highly participate in the deformation accommodation processes [29]. This aspect is supported by a profilometric analysis performed on a Wyko NT 9300 profilometer with a pitch of $5 \mu \mathrm{m}$. Figure 5 presents the outof-plane displacement field obtained after the test which exhibits out-of-plane displacement variations of approximately $20 \mu \mathrm{m}$ over the whole $5 \mathrm{~mm} \times 5 \mathrm{~mm}$ central area. By comparing this field with the microstructure, a more precise analysis shows that the largest displacement gradients are located around grain and twin boundaries where high strain incompatibilities are observed after the test. Consequently, it appears unjustified to smooth the displacement field over grain boundaries as obtained by a classical DIC technique (see Fig. 6, top). This approach

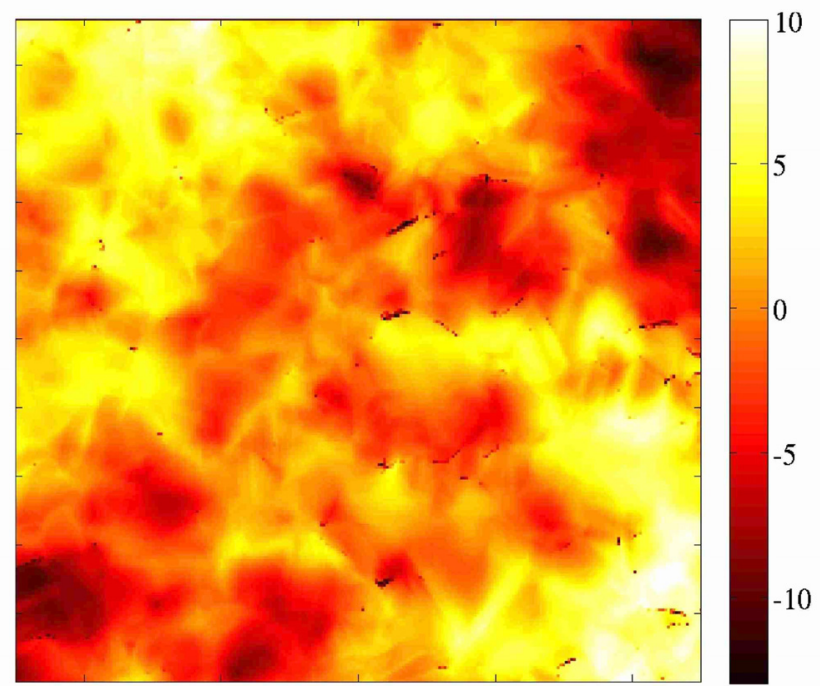

Fig. 5. Profilometric analysis of the out-of-plane displacement field (in $\mu \mathrm{m}$ ) obtained after the test in the $5 \mathrm{~mm} \times 5 \mathrm{~mm}$ central area.

results in a significant underestimation of the localization processes. Here, thermal and kinematic discontinuities will be assumed, as schematically represented at the bottom of the Figure 6 .

This projection operation requires selecting a granular projection basis. This choice is based on different elements: (1) the ability to render the global shape of the observed fields within each grain, (2) its ease of implementation within a least-squares minimization, i.e., a problem with separable variables. In this context, simple polynomial functions are assumed:

$$
\begin{aligned}
A^{g} & =\left(\begin{array}{lllllll}
A_{0} & A_{1} & A_{2} & \ldots & A_{m}
\end{array}\right) \\
N & =\left(\begin{array}{llllllll}
1 & X & Y & X^{2} & Y^{2} & X Y & \ldots & X_{m}
\end{array}\right) \\
F & =\sum_{k=1}^{m} A_{k}^{g} \cdot{ }^{T} N_{k}
\end{aligned}
$$

with $A^{g}$ a set of constants associated within each grain, $N$ the shape function and $F$ the interpolated functions. Taking into account the relative simplicity of such polynomial functions and its ability, in the present case, to render displacement and thermal fields, a full second-degree polynomial function appears to be the most appropriate. The consequences of this second-order polynomial projection technique are the following:

1. Granular treatment: grain boundaries represent a natural discontinuity network within the polycrystal: kinematic and thermal continuities are not ensure on these boundaries. For displacements, this is supported by the profilometric analysis (see Fig. 5) and for temperature, this is justified by studies on the Kapitza effect [30]. Therefore, taking into account grain boundary discontinuities and the proposed treatment provides a better estimation of the local gradients and leads to the definition of a projection domain in which 


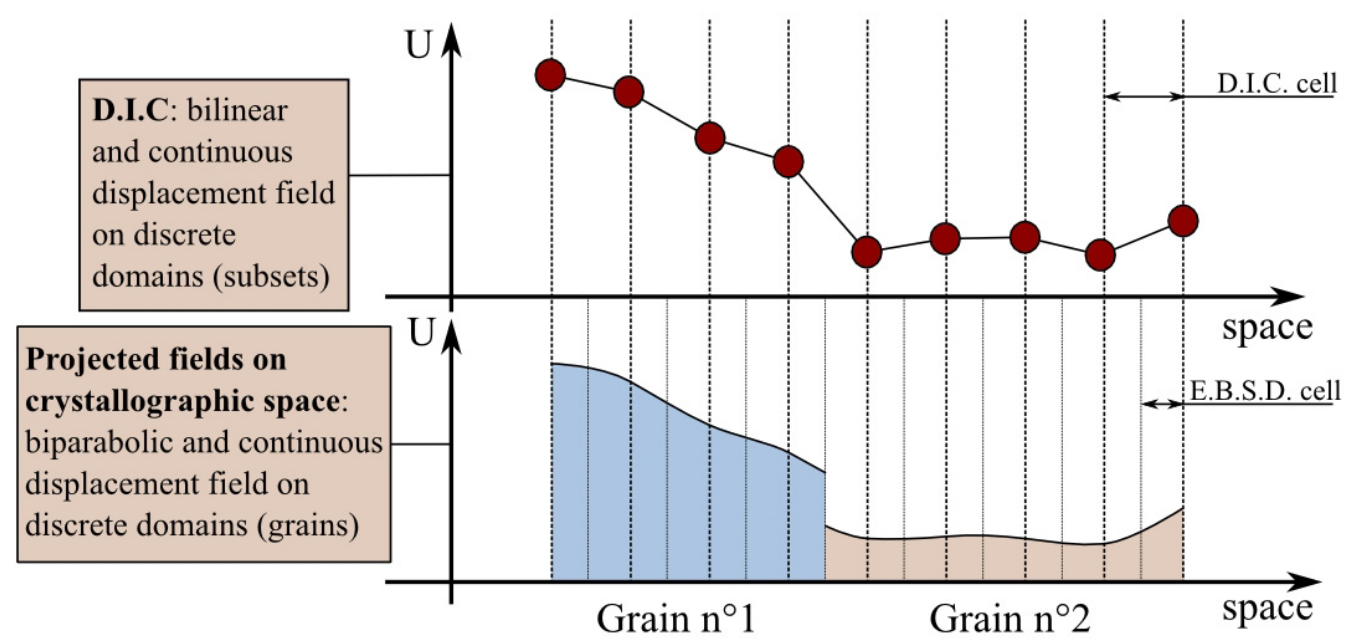

Fig. 6. Schematic of the grain boundary treatment by a DIC technique (at the top) and by assuming field discontinuities (at the bottom).

at best the interpolated functions fit the whole set of intragranular experimental data. Moreover, intragranular quantities can be derived in a simple analytical way.

2. Biparabolic displacement field: in the present case, the main plastic deformation mechanism is the slip activation on a particular slip system. Then displacement fields within each grain and at each time increment are projected on a biparabolic basis and, as strain is calculated from the displacement gradient, we implicitly assume here a bilinear strain field within the grain. As many grains of the $5 \mathrm{~mm} \times 5 \mathrm{~mm}$ central area exhibit a single slip system activation, this assumption is relatively consistent even if it certainly results in greater errors where multiple slip systems activation is observed. The knowledge of the analytic expression of the in-plane displacement field $\underline{u}(x, y)$ within each grain allows an analytic derivation and the local estimation of each plane component of the strain tensor, i.e., $\varepsilon_{11}, \varepsilon_{22}, \varepsilon_{12}$ as well as the in-plane component of the spin tensor $w_{12}$, which can be determined by with $\mathbf{F}=\frac{1}{2}\left(\operatorname{grad}(\underline{u})+{ }^{T} \operatorname{grad}(\underline{u})\right), \varepsilon=\frac{1}{2}\left(\mathbf{F}+{ }^{T} \mathbf{F}\right)$ and $\mathbf{w}=\frac{1}{2}\left(\mathbf{F}-{ }^{T} \mathbf{F}\right)$. Moreover, this assumption also leads to significant variable reduction: six parameters per grain at each moment which represents 12000 variables per field instead of 62500 .

3. Biparabolic thermal field: as conductive fluxes are calculated from the temperature Laplacian, uniform conductive exchanges within the grain at each instant are implicitly assumed through this hypothesis. As every local heat source initiated within the grain is quickly uniform due to the conductive phenomenon, it is therefore assumed that conductive exchanges are instantaneously uniform within the grain and on its boundaries. Then, as for displacement fields, the thermal field can be analytically derived.

It should be pointed out that no data are lost in this projection procedure. The whole decomposition is additive.
Residual fields between initial fields and the projected field are complementary and can be separately analyzed (see Sect. 5). Moreover, in the case of grains with a size lower than the spatial resolutions, fields are projected on lower order polynomial functions. However, one could point out that more than $84 \%$ of the studied area contains grains whose size is greater than the spatial resolution.

\section{Least-squares method}

In order to determine the polynomial parameters set for the entire zone analyzed, a least-squares method is used and, with the same previous notations (see Eq. (3)), one could express this problem as:

$$
\begin{gathered}
E_{A^{g}}=\min _{A^{g}} \sum_{i=1}^{n_{g}}\left[F\left(x_{i}, y_{i}\right)-Z\left(x_{i}, y_{i}\right)\right]^{2} \\
\text { or } \\
E_{A^{g}}=\min _{A^{g}} \sum_{i=1}^{n_{g}}\left[\sum_{k=1}^{m}\left(A_{k}^{g} \cdot{ }^{T} N_{i k}\right)-Z_{i}\right]^{2}
\end{gathered}
$$

with $Z_{i}$ the measured quantity at the position $\left(x_{i}, y_{i}\right)$. Note that the least-squares minimization is performed within each grain ( $A^{g}$ parameter vector). For each coordinate vector $\left(x_{i}, y_{i}\right), i$ ranges from 1 to $n_{g}$, with $n_{g}$ the number of elements within the grain considered, $g$ and $k$ represent the number of parameters to identify; $m=6$ in the case of the biparabolic function. By differentiation, one obtains:

$$
\begin{aligned}
\frac{\partial E}{\partial A^{g}} & =0 \\
2 \sum_{i=1}^{n_{g}} \sum_{k=1}^{6}\left[\left(A_{k}^{g} \cdot{ }^{T} N_{i k}-Z_{i}\right) N_{i k}\right] & =0
\end{aligned}
$$


which leads to a system of six linear equations in $A_{k}^{g}$ that can easily be solved:

$$
\begin{aligned}
& \sum_{i=1}^{n_{g}}\left[\begin{array}{llllll}
1 & x_{i} & y_{i} & x_{i}^{2} & y_{i}^{2} & x_{i} y_{i} \\
x_{i} & x_{i}^{2} & x_{i} y_{i} & x_{i}^{3} & x_{i} y_{i}^{2} & x_{i}^{2} y_{i} \\
y_{i} & y_{i} x_{i} & y_{i}^{2} & y_{i} x_{i}^{2} & y_{i}^{3} & x_{i} y_{i}^{2} \\
x_{i}^{2} & x_{i}^{3} & x_{i}^{2} y_{i} & x_{i}^{4} & x_{i}^{2} y_{i}^{2} & x_{i}^{3} y_{i} \\
y_{i}^{2} & y_{i}^{2} x_{i} & y_{i}^{3} & y_{i}^{2} x_{i}^{2} & y_{i}^{4} & x_{i} y_{i}^{3} \\
x_{i} y_{i} & x_{i}^{2} y_{i} & x_{i} y_{i}^{2} & x_{i}^{3} y_{i} & x_{i} y_{i}^{3} & x_{i}^{2} y_{i}^{2}
\end{array}\right]\left[\begin{array}{c}
A_{0}^{g} \\
A_{1}^{g} \\
A_{2}^{g} \\
A_{3}^{g} \\
A_{4}^{g} \\
A_{5}^{g}
\end{array}\right] \\
& =\sum_{i=1}^{n_{g}}\left[\begin{array}{l}
Z_{i} \\
x_{i} Z_{i} \\
y_{i} Z_{i} \\
x_{i}^{2} Z_{i} \\
y_{i}^{2} Z_{i} \\
x_{i} y_{i} Z_{i}
\end{array}\right]
\end{aligned}
$$

The resolution provides the least-squares-based solution in every point $\left(x_{i}, y_{i}\right)$ within the grain $g$, noted: $Z_{i}=$ $A_{0}^{g}+A_{1}^{g} x_{i}+A_{2}^{g} y_{i}+A_{3}^{g} x_{i}^{2}+A_{4}^{g} y_{i}^{2}+A_{5}^{g} x_{i} y_{i}$.

The least-squares problem is solved in all the grains corresponding to the central $5 \mathrm{~mm} \times 5 \mathrm{~mm}$ area and at each time increment. Figures 7 and 8 present axial strain and thermal fields obtained by the projection procedure. As previously described, the axial strain field is analytically derived from the projected displacement fields. One has to compare both fields with the initial data corresponding to Figures 3 and 4 . Figure 7 shows high strain localization and the slightly deformed areas (dotted lines), which are impossible to distinguish in Figure 3. One can note that certain zones exhibit an axial strain greater than $5 \%$ where the mean value obtained for the complete field at this point is $2.5 \%[19,20]$. In the same way, the assumed thermal localization observed in Figure 4 is clearly underlined in Figure 8 where a conductive front can be seen. This front is located around a highly deformed zone situated at the top of Figure 7 for which many grain boundary incompatibilities are also observed in Figures 2 and 5 . Thermal gradients between grains and inside grains can also be seen. In order to verify this link between thermomechanical fields and microstructural aspects, a particular central grain can be analyzed.

\section{Granular analysis}

The grain presented in Figure 9 is the largest one and is located close to the center of the $5 \mathrm{~mm} \times 5 \mathrm{~mm}$ central area. Its surface is $0.55 \mathrm{~mm}^{2}$ and, for the full-field analysis, it is composed of 54 cells measuring $100 \mu \mathrm{m} \times 100 \mu \mathrm{m}$ (related to the spatial resolutions). A statistical analysis of the slip mark density on the entire micrography shows that the slip mark density in this grain is relatively high and greater than the mean density. The micrography analysis correlated with the EBSD data shows that two systems have been activated in this particular grain (see [31]

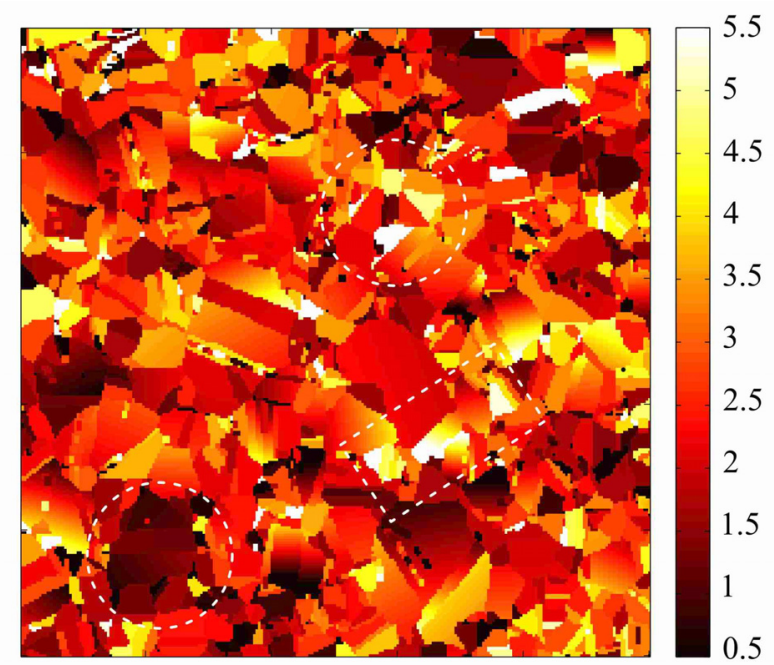

Fig. 7. Axial strain field (in \%) in the $5 \mathrm{~mm} \times 5 \mathrm{~mm}$ central area at the end of the test, during the plastic loading, for which the ratio $\sigma_{22} / R_{e 0.2 \%} \approx 1.2$, obtained from the projected displacement field by an analytical derivation. Within the dotted lines, high strain localization or low deformation can clearly be observed.

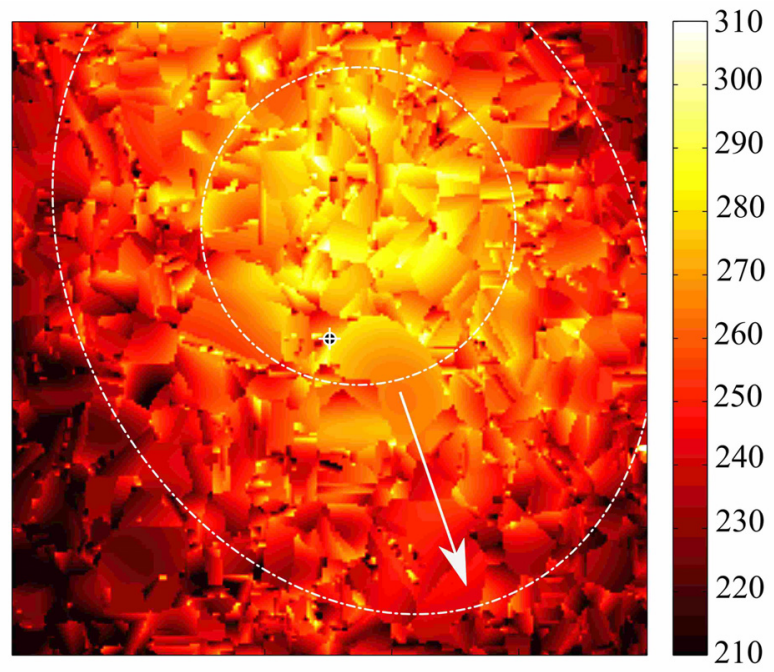

Fig. 8. Projected thermal field (in $\mathrm{mK}$ ) in the $5 \mathrm{~mm} \times 5 \mathrm{~mm}$ central area at the end of the test, during the plastic loading for which the ratio $\sigma_{22} / R_{e .2 \%} \approx 1.2$. The circle represents a conductive front.

for a complete description of crystallography basis): $D_{1}$ with a Schmid factor of 0.339 and $B_{5}$ with a Schmid factor of 0.296 (see Fig. 9). According to the Schmid theory, the $D_{1}$ system should first be activated because of a higher Schmid factor, followed by the $B_{5}$ system. One can also note that the $B_{5}$ system exhibits more intense slip marks. Finally, very intense material discontinuities are observed on its boundaries and four of them correspond to $\Sigma 3$-type boundaries (i.e., the plane for which coincident site lattice/twin is described by a $60^{\circ}$ rotation about $\langle 111\rangle$ planes between neighboring crystallographic domains): top left side, top right side, bottom side and 


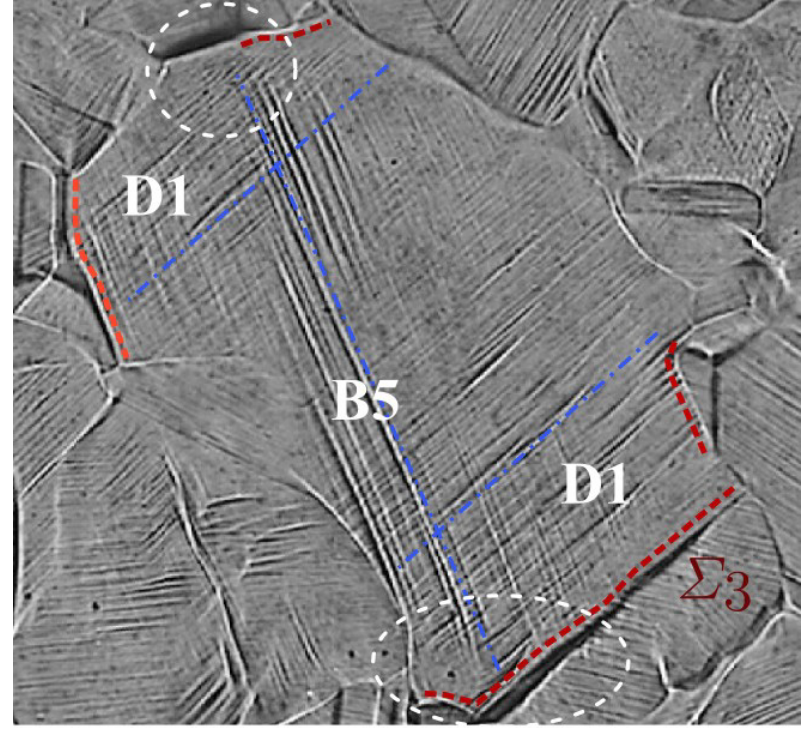

Fig. 9. Micrography of a particular zone, in the center of the $5 \mathrm{~mm} \times 5 \mathrm{~mm}$ central area studied. The largest grain of the entire area can be distinguished. $D 1$ and $B 5$ indicate the activated slip system and the arrows, some zones where considerable grain boundary incompatibilities can be observed.

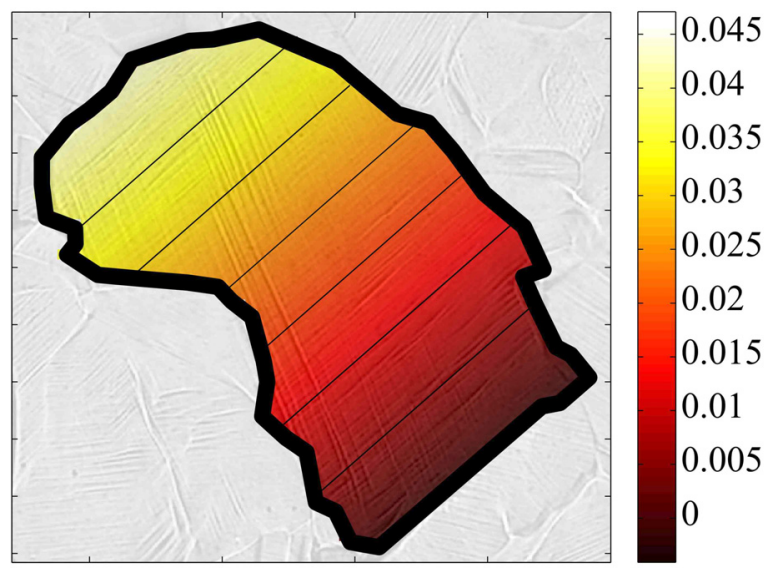

Fig. 10. Intragranular shear strain $\varepsilon_{12}$ (in \%) during the macroscopic elastic response of the specimen. The shear strain gradient is coincident with the $D 1$ slip system orientation. The ratio $\sigma_{22} / R_{e .2 \%} \approx 0.5$.

a small part of the bottom right side (see in Fig. 9). For the first three, significant kinematic discontinuities can be observed after the tensile test on the micrography.

Figures 10, 11 and 12 present intragranular shear strain $\varepsilon_{12}$ and thermal fields at different levels of nominal stress, in the macroscopic elastic or plastic domains $[19,20]$. On the $\varepsilon_{12}$ fields, thin black lines show the isovalues in order to underline the $\varepsilon_{12}$ gradient orientation.

During the macroscopic elastic loading, Figure 10 illustrates that the shear strain gradient is exactly oriented as $D_{1}$ system slip marks. One could consequently affirm

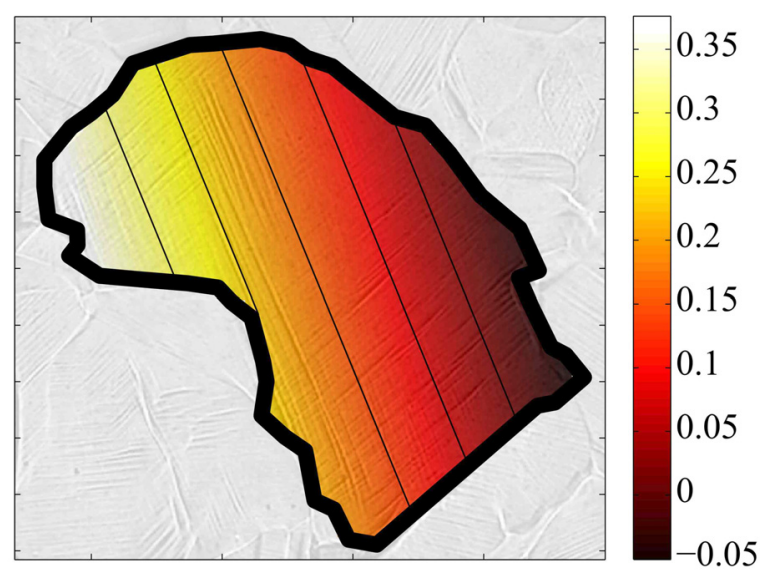

Fig. 11. Intragranular shear strain $\varepsilon_{12}$ (in \%) at the beginning of macroscopic plastic response of the specimen. The shear strain gradient is coincident with the $B 5$ slip system orientation. The ratio $\sigma_{22} / R_{e .2 \%} \approx 1$.

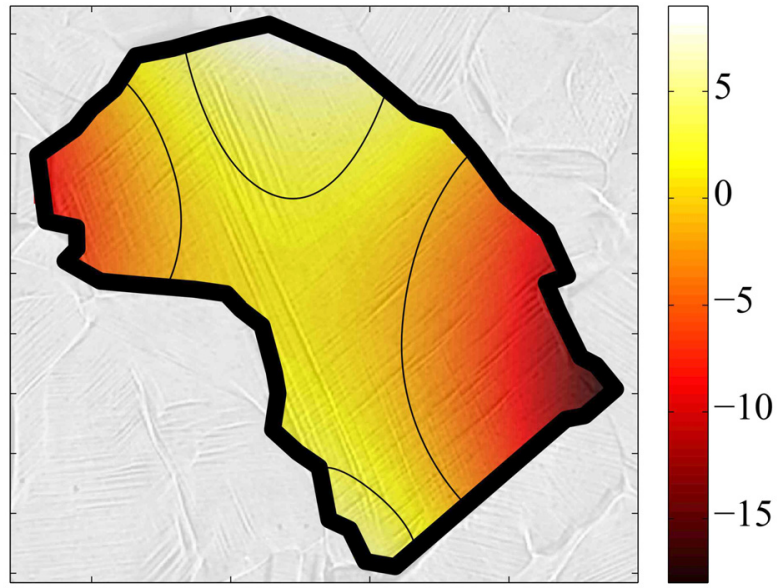

Fig. 12. Intragranular temperature (in $\mathrm{mK}$ ) at the beginning of the macroscopic plastic response of the specimen. The vertical "hot thermal band" is coincident with the $B 5$ slip system orientation and the intense slip marks in Figure 9. The ratio $\sigma_{22} / R_{e .2 \%} \approx 1$.

that at this stress level, which corresponds to a ratio $\sigma_{22} / R_{e 0.2 \%} \approx 0.5$, the $D_{1}$ system is activated. It is in line with the Schmid theory as the Schmid factor of this system is the highest one. Then, comparing Figures 10 and 11 , one can observe that $\varepsilon_{12}$ exhibits a linear gradient that rotates during the test. In Figure 11, which corresponds to a ratio $\sigma_{22} / R_{e .2 \%} \approx 1$, this gradient coincides with the $B 5$ system slip marks and this is the case until the end of the test (not presented here). This rotation is then observed close to the beginning of the macroscopic plastic response. One could therefore conclude that between these two instants, slip activation has changed from the $D_{1}$ system to the $B_{5}$ system. In Figure 11, the shear strain orientation also shows that $B_{5}$ seems to be the only activated slip system during this last period, or 


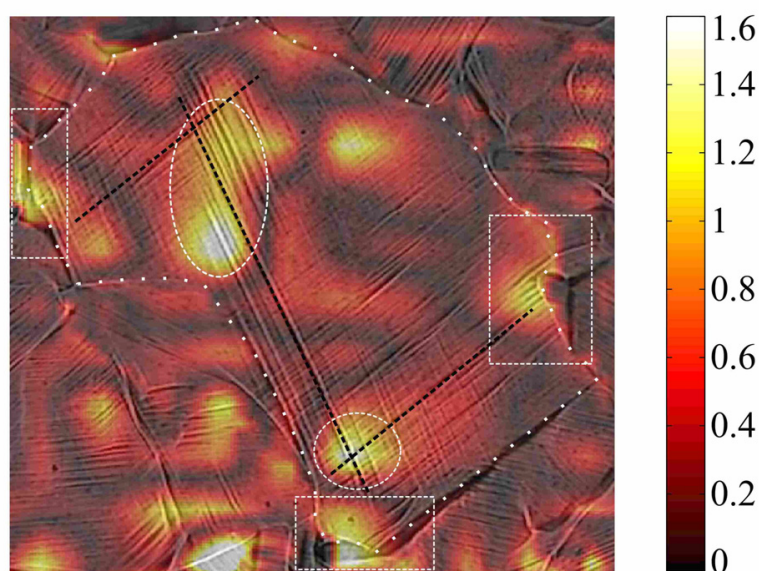

Fig. 13. Intragranular residual axial displacement field (in $\mu \mathrm{m}$ ) in the analyzed grain.

the dominant one, since shear strain isovalues are oriented exactly like the $B_{5}$ system.

Finally, in Figure 12, which corresponds to the same moment as Figure 11, a sort of "hot thermal band" across the grain from top to bottom can be observed. The analysis during the time of the successive pictures of the thermal fields shows that this heat front develops during the rotation of slip systems from $D 1$ to $B 5$. One could note that this "hot band" is in good agreement with the intense $B_{5}$ slip marks observed in Figure 9. Moreover, the hottest points correspond to the extremities of this hot band, i.e., on grain boundaries where intense strain incompatibilities are observed. This illustrates the link between the activation of slip systems and a specific thermal localization.

It has already been pointed out that no data are lost in the projection procedure as the whole decomposition is additive. Residual fields can finally be analyzed and the result shows that their magnitude is relatively low; in the case of the displacement field it corresponds to $1.6 \mu \mathrm{m}$ for the mean value of $500 \mu \mathrm{m}$. It is usefull to focus on the residual displacement field obtained for the previously analyzed grain. Figure 13 shows that the highest level of the residual axial displacement field is obtained in the area where systems $D 1$ and $B 5$ have both been activated. As previously assumed, residual data exhibit the zones where the assumptions are less consistent (multiple activated slip systems, high grain boundary incompatibilities, etc.) even if the values remain totally acceptable compared to the projected full fields. The proposed projection technique seems to be a valuable method to study strain and thermal localization processes in polycrystals.

\section{Conclusion}

This paper was based on the experimental results obtained by Bodelot et al. [20] which propose coupled thermal and kinematic full fields on a $\mathrm{A} 316 \mathrm{~L}$ stainless steel polycristal during a monotonic tensile loading. In order to perform inter- and intragranular thermomechanical analysis at the grain scale in this polycrystal, a crystallography-based projection technique of the temperature and displacement fields on polynomial basis has been proposed. This additive decomposition of the initial data into projected full fields and residual data enables a microstructural analysis of the fields obtained. The application of this method also enables the study of the plastic activity in individual grains. This study shows the pertinence of the method with its intragranular coupled analysis of strain and temperature full fields consistent with the plastic slip activation observed. Further studies are in progress to analyze the beginning of the microplastic behavior at the grain scale and to take subsequent steps towards an energy balance in metallic polycrystals under mechanical loadings.

Acknowledgements. The authors would like to thank Patrick Villechaise at the Institut Pprime - UPR 3346 (ENSMA, Poitiers, France) for the EBSD analysis and fruitful discussions during this study.

The present research work has been supported by International Campus on Safety and Intermodality in Transportation (CISIT) the Nord-Pas-de-Calais Region, the European Community, the Ministry of Higher Education and Research, and the National Center for Scientific Research. The authors gratefully acknowledge the support of these institutions.

\section{References}

[1] G. Sachs, Z. Ver, Zur ableilung einer fleissbedingung, Z. Ver. Dtsch. Ing. 72 (1928) 734-736

[2] E. Schmid, Yield point of a crystals: critical shear stress law, in Proc. 1st Int. Congr. Appl. Mech., Delft, Neetherland, 1924, p. 342

[3] G.I. Taylor, The latent energy remaining in a metal after cold working, Proc. Roy. Soc. London Series A, Containing Papers of a Mathematical and Physical Character 62 (1938) 307-326

[4] A. Cottrell, Dislocations and plastic flow in crystals, Oxford at the Clarendon Press, 1953

[5] C. Barrett, Structure of Metals, McGraw-Hill Book Company, Inc., New York, 1948

[6] W. Boas, M. Hargreaves, On the inhomogeneity of plastic deformation in the crystals of an aggregate, Proc. Roy. Soc. A 193 (1948) 89-97

[7] W.S. Farren, G.I. Taylor, The heat developed during plastic extension of metals. Proc. Roy. Soc. London Series A, Containing Papers of a Mathematical and Physical Character 107 (1925) 422-451

[8] G.I. Taylor, H. Quinney, Proc. Roy. Soc. London Series A, Containing Papers of a Mathematical and Physical Character 143 (1934) 307

[9] H. Quinney, G.I. Taylor, The emission of the latent energy due to previous cold working when a metal is heated. Proc. Roy. Soc. London Series A, Mathematical and Physical Sciences 163 (1937) 157-181

[10] M.B. Bever, D.L. Holt, A.L. Titchener, The stored energy of cold work, Progr. Mater. Sci. 17 (1973) 5-177 
[11] A. Chrysochoos, J.C. Chezeaux, H. Caumon, Analyse thermomécanique des lois de comportement par thermographie infrarouge, Revue de physique appliquée (Paris) 24 (1989) 215-225

[12] J. Hodowany, G. Ravichandran, A. Rosakis, P. Rosakis, Partition of plastic work into heat and stored energy in metals, Experim. Mech. 40 (2000) 113-123

[13] D. Macdougall, Determination of the plastic work converted to heat using radiometry, Experim. Mech. 40 (2000) 298-306

[14] W. Oliferuk, W.A. Swiatnicki, M.W. Grabski, Rate of energy storage and microstructure evolution during the tensile deformation of austenitic steel, Mater. Sci. Eng. A 161 (1993) 55-63

[15] L.M. Clarebrough, M.E. Hargreaves, G.W. West, The release of energy during annealing of deformed metals, Proc. Roy. Soc. A 232 (1955) 252-270

[16] W. Oliferuk, W.A. Swiatnicki, M.W. Grabski, Effect of the grain size on the rate of energy storage during the tensile deformation of an austenitic steel, Mater. Sci. Eng. A 197 (1995) 49-58

[17] C. Badulescu, M. Grédiac, H. Haddadi, J.D. Mathias, X. Balandraud, H.S. Tran, Applying the grid method and infrared thermography to investigate plastic deformation in aluminium multicrystal, Mech. Mater. 43 (2011) 36

[18] A. Saai, H. Louche, L. Tabourot, H. Chang, Experimental and numerical study of the thermo-mechanical behavior of al bi-crystal in tension using full field measurements and micromechanical modeling, Mech. Mater. 42 (2010) 275-292

[19] L. Bodelot, E. Charkaluk, L. Sabatier, P. Dufrénoy, Experimental study of heterogeneities in strain and temperature fields at the microstructural level of polycrystalline metals through fully-coupled full-field measurements by digital image correlation and infrared thermography, Mech. Mater. 43 (2011) 654-670

[20] L. Bodelot, L. Sabatier, E. Charkaluk, P. Dufrénoy, Experimental setup for fully coupled kinematic and thermal measurements at the microstructure scale of an AISI 3161 steel, Mater. Sci. Eng. A 501 (2009) 52-60
[21] L. Allais, M. Bornert, T. Bretheau, D. Caldemaison, Experimental characterization of the local strain field in a heterogeneous elastoplastic material, Acta Metall. Mater. 42 (1994) 3865-3880

[22] F. Lagattu, F. Bridier, P. Villechaise, J. Brillaud, In-plane strain measurements on a microscopic scale by coupling digital image correlation and an in situ SEM technique, Mat. Charac. 56 (2006) 10-18

[23] E. Héripré, M. Dexet, J. Crépin, L. Gélébart, A. Roos, M. Bornert, D. Caldemaison, Coupling between experimental measurements and polycrystal finite element calculations for micromechanical study of metallic materials, Int. J. Plas. 23 (2007) 1512-1539

[24] A.E. Bartali, V. Aubin, S. Degallaix, Fatigue damage analysis in a duplex stainless steel by digital image correlation technique. Fat. Frac. Engng. Mat. Struct. 31 (2007) $137-151$

[25] F. Hild, B. Raka, M. Baudequin, S. Roux, F. Cantelaube, Multiscale displacement field measurements of compressed mineral-wool samples by digital image correlation, Appl. Opt. 41 (2002) 6815-6828

[26] H. Pron, C. Bissieux, Focal plane array infrared cameras as research tools, QIRT J. 1 (2004) 229-240

[27] H. Louche, A. Chrysochoos, Thermal and dissipative effects accompanying lüders band propagation, Mater. Sci. Eng.: A 307 (2001) 15-22

[28] B. Berthel, B. Wattrisse, A. Chrysochoos, A. Galtier, Thermographic analysis of fatigue dissipation properties of steel sheets, Strain 43 (2007) 273-279

[29] L. Priester, Joints de grains et plasticité cristalline, Hermes Science Publications, 2011

[30] C. Nan, R. Birringer, Determining the Kapitza resistance and the thermal conductivity of polycrystals: a simple model, Phys. Rev. B 57 (1998) 8264-8268

[31] A. Kelly, K. Knowles, Crystallography and crystal defects, John Wiley and Sons, 2012 